\title{
Botryoid rhabdomyosarcoma of the nasopharynx
} by

\author{
Virginia C. Canale, Rachele Volpe, $\dagger$ antonino Carbone $\dagger$ and Eligio \\ GRIGOlETto* (Pordenone, and Genova, Italy)
}

\section{Introduction}

Botryoid rhabdomyosarcoma is a common soft-tissue sarcoma of children, the most frequent presentation being in the genito-urinary tract. A less frequent site is the head and neck region involving the pharynx, larynx, maxillary sinus and middle ear (Ragab et al., 1977).

Rhabdomyosarcomas of the head and neck are generally associated with a poor prognosis because of their late diagnosis, inoperability and local recurrences involving especially the base of the skull and meninges. Better definition of the stage of the disease, histology and treatment by the various modern modalities of combined surgery, radiotherapy (RT) and chemotherapy $(\mathrm{CT})$ have significantly altered the prognosis and survival in patients with these tumours, with two-year disease-free survival rates reported as 85 per cent, 83 per cent, 50 per cent, 22 per cent and 6 per cent with Stages I, IIA, IIB, IIIA and IIIB disease respectively (Green and Jaffe, 1978). However, no prognostic correlation or distinction in survival rates has been reported between the botryoid and other variants of embryonal rhabdomyosarcomas of the nasopharynx.

The purpose of this paper is to illustrate a case of Stage III (according to the Intergroup Rhabdomyosarcoma Study) botryoid rhabdomyosarcoma of the nasopharynx associated with a four-and-a-half-year disease-free survival after combined radio-chemotherapeutic treatment.

\section{Case Presentation}

A $121 / 2$-year-old Italian female was admitted to our hospital in January 1974 at six years of age with signs of nasal obstruction and sinusitis of increasing intensity during the two months preceding hospitalization. No other systemic symptoms were evident. Since the age of six months, the child had experienced frequent episodes of mucopurulent rhinitis and asthmatic bronchitis. ENT examination revealed mucopurulent material in both nasal fossae, several sessile polypoid masses occupying the nasopharynx, and bilateral diffuse adenopathy of the submandibular and lateral cervical lymph nodes. No other signs of metastatic disease were apparent.
Initial haematological, biochemical and urinary evaluations, including a 24-hour VMA determination, were within normal limits. A chest $\mathrm{X}$-ray was normal. Sinus $\mathbf{X}$-rays and tomograms of the upper airways demonstrated opaque masses occupying the nasal fossae with extension into the right maxillary sinus. Histological examination of a nasopharyngeal mass biopsy revealed embryonal rhabdomyosarcoma.

No further surgery was undertaken and the patient was treated with 6,000 rads ${ }^{60}$ cobalt radiotherapy (TCT) in 30 sessions to the nasopharynx and maxillary sinuses, with greater than 75 per cent reduction of the tumour masses.

Post-TCT, the patient did well until January 1975, when one right and one left enlarged, firm,

* Division of Oncology and Radiotherapy, Ospedale Civile, Via Montereale, 33170, Pordenone, Italy.

$\dagger$ Department of Pathology, Istituto Scientifico per Lo Studio e La Cura di Tumore, Istituto di Oncologia dell'Università, Viale Benedette XV $\mathrm{XV}^{\circ}, 10,16132$, Genova, Italy. 
fixed submandibular lymph nodes were noted, measuring $2 \times 1.5 \mathrm{~cm}$. and $6 \times 6.5 \mathrm{~cm}$. respectively. Re-evaluation studies, including a bone marrow aspiration, lumbar puncture and pulmonary tomograms, were all negative except for an opacification of the soft tissue and a mild compression of the left hypopharynx. A left lymph node biopsy histologically confirmed the presence of metastasis. CT with five daily doses of Adriamycin $\left(10 \mathrm{mg} / \mathrm{m}^{2}\right.$.) was administered followed by 4,000 rads TCT in 20 sessions to both antero-lateral lymph node chains.

From July to December 1975, following the completion of TCT, the patient was treated with six-monthly cycles of intravenous CT according to Gottlieb et al. (1972): Adriamycin $50 \mathrm{mg}$. Day 1, Cyclophosphamide $500 \mathrm{mg}$. Day 1, Vincristine $1.2 \mathrm{mg}$. Days 1 and 5 and DTIC $300 \mathrm{mg}$. Days 1 to 5. The patient tolerated CT with no complications. A right maxillary sinus biopsy in February 1976 revealed no evidence of malignancy.

The patient has maintained a four-and-a-halfyear complete remission since suspension of CT or a six-and-a-half-year survival since diagnosis. Consequences of RT include partial atrophy of the nasal and nasopharyngeal mucosa, multiple dental caries and enamel dysplasia, minimal atrophy of the maxillary bones, and bilateral persistent opacification of the maxillary sinuses.

\section{Pathological Findings}

Grossly, the initial biopsy from the nasopharyngeal tumour appeared as a translucent, gelatinous, grape-like mass. This $3 \times 2 \mathrm{~cm}$. polypoid mass was soft, shiny and partially ulcerated.

Microscopically, the neoplasm was primarily composed of immature cells with a loose structure. The tumour showed a relatively dense layer of less differentiated, round or spindle rhabdomyoblastic cells of small and medium size, three to five cells thick, with many mitoses. The surface layer was covered with benign surface epithelial cells of the respiratory mucosa (Fig. 1). Immediately beneath the layer of rhabdomyoblasts, there was abundant myxoid loose tissue containing small blood vessels, stellate cells and several small rounded and elongated cells with acidophilic cytoplasm containing two or more nucleoli and a moderate amount of mitotic activity. Features suggestive of cross-striation could not be observed in their cytoplasm.

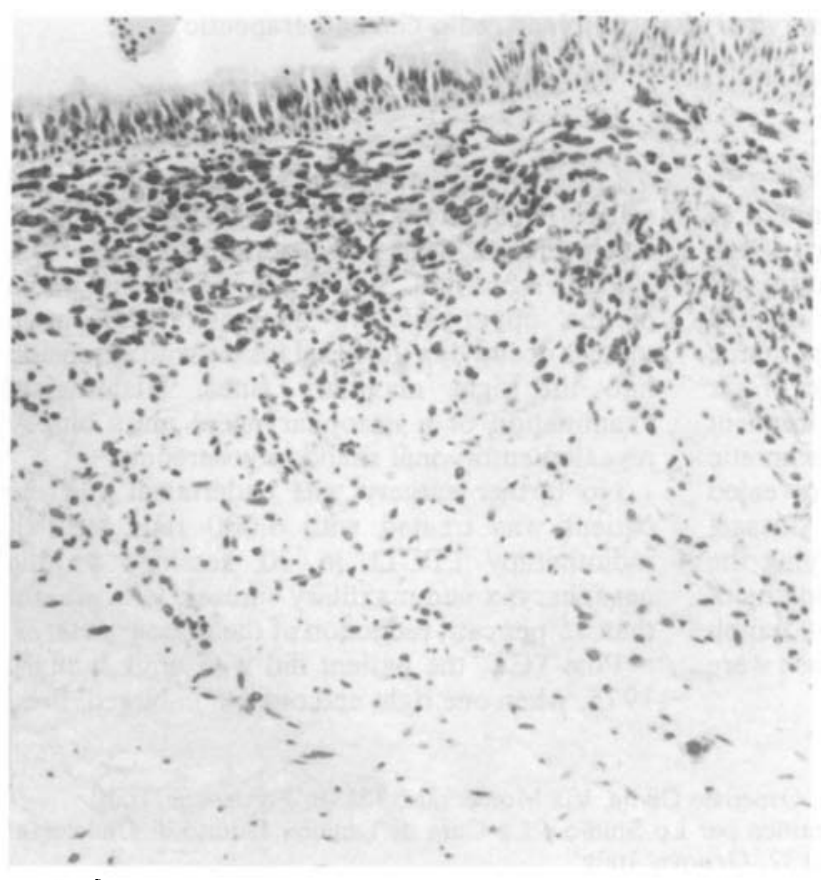

\section{FIG. 1}

Botryoid rhabdomyosarcoma. The subepithelial zone contains embryonal rhabdomyoblasts as well as myxoid loose tissue. Haematoxylin and Eosin $\times 130$. 
The histologic diagnosis was embryonal rhabdomyosarcoma of loose or botryoid type according to the International Society of Pediatric Oncology (SIOP) classification (Gerard-Marchant et al., 1978). The histologic examination of a lymph node biopsy performed one year after the initial diagnosis revealed unequivocal metastatic lesions by embryonal rhabdomyosarcoma, whereas the control bone marrow aspirates and spinal fluid cytological examinations were always negative.

\section{Discussion}

The recognition of sarcoma botryoides as a distinct clinical and pathological entity, and the description of alveolar rhabdomyosarcoma complete the frequently-used grouping of rhabdomyosarcomas into embryonal, botryoid, alveolar and pleomorphic types (Horn and Enterline, 1958). However, the histologic classification adopted by SIOP differentiated two main types of rhabdomyosarcomas: embryonal and adult (Gerard-Marchant et al., 1978). The former included the loose (or botryoid), dense, myoblastic and alveolar (Riopelle's) rhabdomyosarcomas.

Embryonal rhabdomyosarcoma is a common soft-tissue sarcoma of children with a median age incidence of five years (Green and Jaffe, 1978). Although this tumour can develop in any area of the body, the botryoid variant occurs near the mucosal surface (Stout and Lattes, 1967).

Clinical manifestations of tumours of the nasopharynx and paranasal sinuses include local pain and swelling, nasal obstruction, nasal discharge, epistaxis and sinusitis. The overall incidence of positive regional lymph nodes at presentation for rhabdomyosarcomas of the head and neck is estimated to be 3 per cent (Green and Jaffe, 1978). Local recurrences with direct meningeal extension or distant metastases to the lymph nodes, lungs, bones, liver and bone marrow tend to develop within an average of 7.6 months, with approximately 70 per cent occurring within one year from diagnosis (Ragab et al, 1977).

The value of combined surgery, CT and RT has been clearly demonstrated in several studies (Ghavimi et al., 1975; Donaldson et al., 1973; Mauer et al, 1977). Surgical excision, whenever possible, is recommended for head and neck tumours but should not involve major loss of function or cosmetic deformity. Current recommendations for RT are 5,000-6,000 rads
TCT to the primary tumour, resulting in local control in 80-90 per cent of non-orbital head and neck tumours (Ragab et al., 1977). Combination CT with VAC and RT have proved extremely efficacious, especially in inoperable tumours. Donaldson et al. (1973) achieved a local control rate of 89 per cent. Of these patients, 74 per cent were free of disease two to seven years later, with a median disease-free interval of two years eight months.

Several factors, including extent of disease, site, histologic type and age modify the prognosis (Pratt et al, 1978). Rhabdomyosarcomas of the head and neck, excluding the orbit, are presently associated with a 68-75 per cent disease-free survival (Green and Jaffe, 1978). The best survivals regarding histologic type have been reported with sarcoma botryoides (site not specified), although in the National Rhabdomyosarcoma Study, the pathologic type did not affect the survival rate (Ragab et al., 1977). Children under seven years of age have a significantly better survival than older children due to less extensive disease at the time of diagnosis, lower rates of recurrence and a more favourable histologic type.

Our patient appears to have met all these favourable criteria and therefore the long-term disease-free survival was expected but still noteworthy since it is above the median reported in the literature for the stage of the disease. This may be due in part to its histologic type and in part to the combined therapy employed. We suggest that histologic type and prognosis of rhabdomyosarcomas of the nasopharynx may be better correlated in future studies of patients.

\section{Summary}

A case report of a Stage III botryoid rhabdomyosarcoma of the nasopharynx associated with a six-and-a-half-year survival is presented. Treatment consisted of surgery, radiotherapy $(6,000$ rads TCT) to the nasopharynx and maxillary sinuses bilaterally, and six cycles of polychemotherapy with Vincristine, Adriamycin, Cyclophosphamide and DTIC, without major loss of function or cosmetic deformity. The histology of the lesion is discussed with reference to recent classification and prognosis. The authors suggest that the histological type and prognosis of rhabdomyosarcoma of the nasopharynx in children may be better correlated in future studies. 


\section{References}

Donaldson, S. S., Castro, J. R., Wilbur, J. R, and Jesser, R. H. JR. (1973) Rhabdomyosarcoma of head and neck in children. Cancer, 31: 26.

Gerard-Marchant, R, Marsden, H. B., and VAn UnNik, A. J. M. (1978) Histological classification of rhabdomyosarcomas. In Abstracts, Xth Meeting of the International Society of Pediatric Oncology (SIOP), Brussels, Belgium. 20-23 September, p. 13.

Ghavimi, P., Exelby, P. R, and D'ANgio, G. I. (1975) Multidisciplinary treatment of embryonal rhabdomyosarcoma in children. Cancer, 35: 677.

Gotrtleb, J. A., BaKeR, L. H., and QuAglianA, J. M. (1972) III. Chemotherapy of sarcomas with a combination of Adriamycin and dimethyl triazeno imidazole carboxamide. Cancer, 30: 1632.

Green, D. M., and JAFFe, N. (1978) Progress and controversy in the treatment of childhood rhabdomyosarcoma. Cancer Treatment Reports, 5: 7.

HORN, R C. JR, and ENTERLINE, H. T. (1958) Rhabdomyosarcoma: a clinicopathological study and classification of 39 cases. Cancer, 11: 181 .

Mauer, H. M., Moon, T., and Donaldson, M. (1977) Preliminary results of the Intergroup Rhabdomyosarcoma Study (IRS). In Management of Primary Bone and Soft Tissue Tumors. Year Book Medical Publishers, Inc., p. 317.

Pratt, C. B., Smith, J. W., and Woerner, S. (1978) Factors leading to delay in the diagnosis and affecting survival of children with head and neck rhabdomyosarcoma. Pediatrics, 61: 30.

Ragab, A. A., Phelan, E. T., and Razek, A. A. (1977) Malignant tumors of the soft tissues. Clinical Pediatric Oncology. Eds. W. W. Sutow, T. J. Vietti, and D. J. Fernback. The C. V. Mosby Co., St. Louis, p. 569.

Stout, A. P., and Latres, R (1967) Tumors of the soft tissues. Atlas Tumor Pathology. 2nd. Series, Fasc. 1. Armed Forces Institute of Pathology, Washington, DC, p. 138.

Address for reprints:

Dr. V. C. Canale,

Divisione di Radioterapia e Oncologia Medica,

Ospedale Civile di Pordenone,

Via Montereale,

33170,

Pordenone,

Italia. 